\title{
A SINGULAR PERTURBATION SOLUTION TO A PROBLEM OF EXTREME TEMPERATURES IMPOSED AT THE SURFACE OF A VARIABLE- CONDUCTIVITY HALFSPACE : SMALL SURFACE CONDUCTIVITY*
}

\author{
By
}

LEONARD Y. COOPER

Bell Telephone Laboratories, Incorporated, Whippany, N. J.

\begin{abstract}
The transient temperature field resulting from a constant and uniform temperature $T_{s}$ (or time-dependent heat flux $H=h t^{-1 / 2}$ ) imposed at the surface of a halfspace initially at uniform temperature $T_{0}$ is considered. A temperature-dependent thermal conductivity variation, $k(T)=k_{0} \exp \left[\lambda\left(T-T_{0}\right) / T_{0}\right]$, and a constant product of density and specific heat, $\rho C$, are assumed to be accurate models for the halfspace for some useful temperature range. The problem is initially formulated in terms of the dimensionless conductivity $\phi=k(T) / k_{0}$. Attention is then focused on the singular problem resulting from the limits $\phi_{s}=\phi\left(T_{s}\right) \downarrow 0$ and $\phi_{s} \rightarrow \infty$. This work considers the use of matched asymptotic expansions to solve the problem under the first of these limits. In particular, Fraenkel's interpretation [5] of Van Dyke's method of inner and outer expansions [6] is carefully applied to the problem under consideration. Besides obtaining a uniformly valid solution to the problem, a particularly interesting explicit result is deduced, namely
\end{abstract}

$$
\lim _{\phi_{s} \downarrow 0} h=-(1.182754 \cdots)\left(T_{0} / \lambda\right)\left[\rho C k_{0} / 2\right]^{1 / 2}+O\left(\phi_{s} \ln \phi_{s}\right) .
$$

\section{Introduction.}

1.1 The problem. This work is concerned with the solution for the transient temperature field $T(x, t)$ in a halfspace initially at uniform temperature $T_{0}$, where at time $t=0$ a temperature $T_{s} \neq T_{0}$ is imposed and maintained at the free surface $x=0$. As in the example problem of [1], a temperature-dependent thermal conductivity variation,

$$
k=k(T)=k_{0} \exp \left[\lambda\left(T-T_{0}\right) / T_{0}\right],
$$

and a constant product of density and specific heat, $\rho C$, are assumed as accurate models for the halfspace for some useful temperature range. $\lambda$ may be positive or negative. This problem (with different interpretations for $T$, $k$, etc.) has also been studied in [2], [3] and [4]. In those works results of the solution field for several values of $k\left(T_{s}\right) / k_{0}$ have been obtained.

In [1] we considered the use of regular perturbation expansions of the temperature (or conductivity) field about its initial state. Here we are concerned with a singular

* Received March 1, 1973; revised version received September 27, 1973. This work was supported by the U. S. Army under Contract DAHC-60-71-C-0005. 
perturbation solution to the problem when arbitrarily small or large surface conductivities (i.e. when arbitrarily large (hot) or small (cold) surface temperatures) are imposed. After formulating the problem under such limiting conditions we will study the solution under the former small-surface-conductivity limit. Results from [1] for moderate surface conductivity (i.e., moderate $\left.\lambda\left(T_{s}-T_{0}\right) / T_{0}\right)$ and from the present work for arbitrarily small $k\left(T_{s}\right)$ will be seen to have common regions of applicability.

The boundary condition on surface temperature could be replaced by an appropriate boundary condition on surface heat transfer rate, $H(t)$. As pointed out in [1], such a (presumably unique) boundary condition is

$$
H(t)=-k(T) \partial T / \partial x=h t^{-1 / 2}, \quad x=0,
$$

where $h$, a constant, is a measure of the "amplitude" of the heat transfer rate to the material halfspace. This proportionality of $H$ to $t^{-1 / 2}$ is, in fact, valid for any temperature-dependent conductivity material (with $\rho C$ constant).

1.2 The governing equations and some remarks on the solution. It is convenient to study the problem in dimensionless variables. Furthermore, for our assumed conductivity variation with temperature, a dimensionless conductivity $\phi$ is a convenient dependent variable. Thus we define

$$
V=\lambda\left(T-T_{0}\right) / T_{0} ; \quad \phi=k(T) / k_{0}=\exp V .
$$

We also define a dimensionless independent (similarity) variable $\eta$, the dimensionless ("amplitude" of heat transfer) parameter $\zeta$, and the dimensionless surface conductivity $\phi_{s}$, by

$$
\begin{aligned}
\eta & =x\left[2 t k_{0} / \rho C\right]^{-1 / 2}, \\
\zeta & =-\lambda h\left[\rho C k_{0} / 2\right]^{-1 / 2} / T_{0}, \\
\phi_{s} & =\exp V_{s}=\exp \left[\lambda\left(T_{s}-T_{0}\right) / T_{0}\right] .
\end{aligned}
$$

In line with earlier remarks, the variable $\phi=\phi(\eta)$ can be considered as having parametric dependence on $\phi_{s}$ or on $\zeta$ with some a priori unknown functional relationship between these two parameters. Indeed, obtaining this relationship for any $\phi_{s}>0$ will be one of the most significant results of this investigation.

The boundary-value problem governing $\phi$ is [1]

$$
\begin{gathered}
\phi d^{2} \phi / d \eta^{2}+\eta d \phi / d \eta=0 ; \\
d \phi(0) / d \eta=\zeta \quad \text { or } \quad \phi(0)=\phi_{s}, \quad \text { and } \phi(\infty)=1 .
\end{gathered}
$$

In [1] different expansions of $\phi$ about the $\phi=1$ state were considered. It was concluded that the $\zeta$ expansion of $\phi$ given by

$$
\phi=1+\sum_{n=1}^{\infty} \zeta^{n} \phi_{n}{ }^{(3)}(\eta),
$$

where in actual computation the above series was truncated after three terms, gave a useful analytic approximation to the solution function over a significant range of the $\zeta$ or $\phi_{s}$ parameter.

As mentioned earlier, we will presently concern ourselves with the problem for limiting small and large values of $\phi_{s}$, i.e. arbitrarily large values of $\left|V_{s}\right|$. Using the 
solution to such a problem together with the results from [1] given, say, by using the three-term approximation to the series of Eq. (6), one would hope to deduce a useful representation of the solution to our problem for arbitrary positive values of $\phi_{s}$.

1.3 Restatement of the boundary-value problem. For the present purpose it is convenient to change our two-point boundary-value problem into an initial-value problem. This can be accomplished by considering the solution to the one-parameter problem

$$
\begin{gathered}
\psi d^{2} \psi / d z^{2}+z d \psi / d z=0, \quad-\infty<z<\infty, \\
d \psi / d z=1 \text { and } \psi=\psi_{s} \text { at } z=0 .
\end{gathered}
$$

We assume the solution, $\psi\left(z ; \psi_{s}\right)$, to the above problem has been obtained for all $z$ and for arbitrary positive $\psi_{s}$. Consistently with the above statement of the problem for $\psi$, we also assume that such a solution has positive asymptotic values $\psi\left(\infty, \psi_{s}\right)>\psi_{s}$ and $\psi\left(-\infty, \psi_{s}\right)<\psi_{s}$ as $z \rightarrow \infty$ and $z \rightarrow-\infty$ respectively. Then the solution can be transformed to our desired $\phi$ solution to Eqs. (5) as follows:

For $\zeta>0$ or $\phi_{s}<1$ define

$$
\zeta=\psi^{-1 / 2}\left(\infty ; \psi_{s}\right) ; \quad \eta=z \zeta ; \quad \phi=\psi\left(z ; \psi_{s}\right) \zeta^{2}, \quad z \geq 0 .
$$

For $\zeta<0$ or $\phi_{s}>1$ define

$$
\zeta=-\psi^{-1 / 2}\left(-\infty, \psi_{\mathrm{s}}\right) ; \quad \eta=z \zeta ; \quad \phi=\psi\left(z ; \psi_{\mathrm{s}}\right) \zeta^{2}, \quad z \leq 0 .
$$

We will concern ourselves in what follows with the solution to the problem for $\psi$ as stated in Eqs. (7) and (8).

1.4 Solution for $\psi$ for large $\psi_{s}$. A careful study of Eqs. (7) and (8) reveals that in the limit $\psi_{s} \rightarrow \infty$ our problem is identical to the regular perturbation problem treated in [1]. In fact, considering the transformation

$$
\bar{\phi}=\psi / \psi_{s}+\sum_{n=1}^{\infty} \bar{\zeta}^{n} \phi_{n}{ }^{(3)}(0), \quad \bar{\zeta}= \pm \psi_{s}^{-1 / 2}, \quad \bar{\eta}= \pm z \psi_{s}^{-1 / 2}
$$

where top and bottom signs are for $z \geq 0$ and $z \leq 0$ respectively and where the functions $\phi_{n}{ }^{(3)}(\eta)$ have been reintroduced from [1] in our present Eq. (6), we find that the problem for $\bar{\phi}$ is given by

$$
\begin{gathered}
\bar{\phi} d^{2} / d \bar{\eta}^{2}+\bar{\eta} d \Phi / d \bar{\eta}=0, \quad \bar{\eta} \geq 0 ; \\
d \bar{\phi} / d \bar{\eta}=\bar{\zeta}, \quad \bar{\phi}=1+\sum_{n=1}^{\infty} \bar{\zeta}^{n} \phi_{n}{ }^{(3)}(0) \quad \text { at } \quad \bar{\eta}=0 .
\end{gathered}
$$

Here the limit $\psi_{s} \rightarrow \infty$ corresponds to the limit $\bar{\zeta} \rightarrow 0$. But the $\zeta$ expansion representation for $\phi$ of Eq. (6) which has been studied in [1] satisfies both the differential equation and the boundary conditions of Eqs. (12). If we assume that the solution to our problem is unique and that the expansion has a finite radius of convergence $\left|\zeta_{R}\right|$ (for all nonnegative $\eta$ ) in the complex $\zeta$ plane, then within that radius of convergence the solution for $\bar{\phi}$ is identical to the solution for $\phi$. Moreover, the $\zeta$ expansion solution for $\phi$, i.e. the $\phi_{n}{ }^{(3)}(\eta)$, has been obtained in [1], an explicit analytic representation for the first three of these functions being given in Eqs. (33), (35), and (36) of that work.

In view of the above discussion we can dismiss the limit $\psi_{s} \rightarrow \infty$ for now and concentrate on the limit $\psi_{s} \downarrow 0$.

1.5 Solution for $\psi$ for limiting small $\psi_{s} ;$ some observations. In this and later sections 
we will be considering solutions for $\psi$ when $\psi_{s} \downarrow 0$. To follow the development more easily we introduce the small parameter designation $\epsilon$ and use it interchangeably with $\psi_{s}$, i.e., $\epsilon \equiv \psi_{s}$.

An investigation of the initial-value problem for $\psi$ given by Eqs. (7) and (8) reveals that for the limit $\epsilon \equiv \psi_{s} \downarrow 0$ the solution involves a singular perturbation in the neighborhood of $z=0$. In other words, in the sense discussed by Fraenkel [5] there is no single asymptotic expansion of the form

$$
\psi \sim \sum_{n=0}^{N} G_{n}(\epsilon) \psi_{n}(z)
$$

where $G_{n+1}=o\left(G_{n}\right)$ for $\epsilon \downarrow 0$, which describes $\psi$ with an error which is $o\left(G_{N}\right)$ in the entire interval $-\infty<z<\infty$ or even in some fixed, bounded neighborhood of $z=0$. That this is so becomes obvious, for example, from the following considerations.

Assume that $\psi$ can indeed be described by an expansion of the above form in some fixed, bounded neighborhood of $z=0$. If we use this expansion in Eq. (7), impose the initial conditions of Eq. (8) and take the limit as $\epsilon \downarrow 0$, it becomes apparent that we could take $G_{0}=1$ without loss of generality. Then $\psi_{0}=z+0\left(z^{2}\right)$. Now, for a nontrivial $\psi_{1}$ and in order that the condition $\psi(0 ; \epsilon)=\epsilon$ of Eq. (8) be satisfied we can, again without loss of generality, choose $G_{1}=\epsilon$. Then

$$
d^{2} \psi_{1} / d z^{2}+\left(z / \psi_{0}\right)\left[d \psi_{1} / d z-\left(1 / \psi_{0}\right)\left(d \psi_{0} / d z\right) \psi_{1}\right]=0,
$$

$$
\psi_{1}=1, \quad d \psi_{1} / d z=0 \text { at } z=0 .
$$

Using the above result for $\psi_{0}$ and the boundary conditions for $\psi_{1}$ in the above equation for $\psi_{1}$ reveals that $\lim d^{2} / \psi_{1} / d z^{2}=1 / z+o(1 / z)$, as $z \rightarrow 0$. Without proceeding further or in greater depth it is apparent that this last result is inconsistent with the boundary condition, $d \psi_{1} / d z=0$ at $z=0$. The problem is that if our earlier expansion form for $\psi$ is taken as valid for arbitrarily small $\epsilon$ then it is not uniformly valid for $z \rightarrow 0$. It is for this reason that we cannot frecly require boundary conditions at $z=0$ to be satisfied by the assumed asymptotic representation for $\psi$ even though for fixed nonzero $z$ the expansion may be a suitable representation of our solution function for limiting small values of $\epsilon$. The actual boundary conditions for the $\psi_{n}(z)$ and the proper choices for the $G_{n}(\epsilon)$ must be deduced from a solution representation which is uniformly valid at $z=0$. Such a solution representation can be obtained in the stretched variable $\hat{z}=z / \epsilon$. As we will see, considerations of $\psi=\epsilon \hat{\psi}(\hat{z} ; \epsilon)$ together with $\psi(z ; \epsilon)$ will allow for a $\psi$ solution uniformly valid for limiting small positive values of $\epsilon$ and for fixed $z / \epsilon>-1$. A uniformly valid solution for small positive $\epsilon$ and for arbitrary $z \leq 0$ will require further coordinate expansion considerations. Such a solution will be the objective of another investigation.

2. Solution for $\psi$ in the limit $\epsilon \downarrow 0$ for $z>-\epsilon$.

2.1 Some assumptions and the matching principle. In view of the concluding remarks of the last section we define

$$
\hat{z}=\hat{y}-1=z / \epsilon, \quad \hat{\psi}=\psi / \epsilon .
$$

We designate $\hat{z}$ as our inner variable and $z$ as our outer variable, and rewrite the initialvalue problem for $\psi$ in the $\hat{\psi}, \hat{y}$ variables. Thus

$$
\hat{\psi} d^{2} \hat{\psi} / d \hat{y}^{2}+\epsilon(\hat{y}-1) d \hat{\psi} / d \hat{y}=0, \quad \hat{\psi}(1)=d \hat{\psi}(1) / d \hat{y}=1 .
$$


Assumption 1. We now assume that under the inner limiting process

$$
\epsilon \downarrow 0 \text { with } \hat{y} \text { fixed in an interval } \hat{y}_{0}<\hat{y}<\hat{y}_{1}
$$

(for some fixed $\hat{y}_{0}<1$ and $\hat{y}_{1}>1$, both independent of $\epsilon$ ), $\psi$ has an inner expansion to $M+1$ terms of the form

$$
\psi=\epsilon \hat{\psi}=\sum_{m=0}^{M} g_{m}(\epsilon) \hat{\psi}_{m}(\hat{y})+O\left[g_{M+1}(\epsilon)\right] .
$$

We allow $M$ to be any nonnegative integer and the $\left\{g_{m}\right\}(m=0,1, \cdots, M+1)$ to be an appropriate sequence of gauge functions such that $g_{m+1}=o\left(g_{m}\right)$ as $\epsilon \downarrow 0$. We further denote the series in Eq. (16) as $H_{M}(\psi)$. Thus

$$
H_{M}(\psi)=\sum_{m=0}^{M} g_{m}(\epsilon) \hat{\psi}_{m}(\hat{y}) .
$$

Assumption 2. In a way similar to the above we assume that under the outer limiting process

$$
\epsilon \downarrow 0 \text { with } z \text { fixed in the interval } 0<z_{0} \leq z<\infty
$$

(where $z_{0}$ is an arbitrarily small positive number, but independent of $\epsilon$ ), $\psi$ has an outer expansion to $N+1$ terms of the form

$$
\Psi=\sum_{n=0}^{N} G_{n}(\epsilon) \psi_{n}(z)+O\left[G_{N+1}(\epsilon)\right] .
$$

We allow $N$ to be any nonnegative integer and the $\left\{G_{n}\right\}(n=0,1, \cdots, N+1)$ to be an appropriate sequence of gauge functions such that $G_{n+1}=o\left(G_{n}\right)$ as $\epsilon \downarrow 0$. Finally, we denote the series of Eq. (19) as $E_{N}(\psi)$. Thus

$$
E_{N}(\psi)=\sum_{n=0}^{N} G_{n}(\epsilon) \psi_{n}(z)
$$

Note that whereas the gauge functions $\left\{g_{m}\right\}$ and $\left\{G_{n}\right\}$ introduced in the above assumptions are not unique, neither are they arbitrary. In fact, they must be complete enough to allow Eqs. (16) and (19) to be valid. As we will see, we can develop both an appropriate sequence $\left\{g_{m}\right\}$ and the corresponding $\hat{\psi}_{m}(\hat{y})$, compatible with Eq. (16), directly from the initial-value problem as stated in Eq. (14). As for obtaining a sequence $\left\{G_{n}\right\}$ and corresponding $\psi_{n}(z)$ compatible with Eq. (19), these must be deduced from Eq. (7) with the use of some valid principle for matching the inner and outer expansions of $\psi$. Such a matching principle will supply the conditions (lacking for want of the usual initial or boundary conditions) required to solve for $\psi$ in outer variables (i.e., required to solve Eq. (7)). Here we will assume Van Dyke's matching principle [6] to be valid. We utilize Fraenkel's interpretation of this principle [5] which, for our unknown function $\psi$, we state as follows:

Matching PRINCIPLe. If assumptions 1 and 2 are correct for some sequence $\left\{g_{n}\right\}$ $(n=0,1, \cdots, N+1)$ and some sequence $\left\{G_{m}\right\}(m=0,1, \cdots, M+1)$ then: (a) under the outer limiting process defined in (18) the function $H_{M}(\psi)$ has an outer expansion to $N+1$ terms of the form

$$
H_{M}(\psi)=\sum_{n=0}^{N} G_{n}(\epsilon) \mathcal{H}_{n}{ }^{(M)}(z)+O\left(G_{N+1}\right)
$$


where we denote the series of Eq. (21) as $E_{N}\left[H_{M}(\psi)\right]$. Thus

$$
E_{N}\left[H_{M}(\psi)\right]=\sum_{n=0}^{N} G_{n}(\epsilon) \mathfrak{H}_{n}{ }^{(M)}(z) .
$$

(b) Under the inner limiting process defined in (15) the function $E_{N}(\psi)$ has an inner expansion to $M+1$ terms of the form

$$
E_{N}(\psi)=\sum_{m=0}^{M} g_{m}(\epsilon) \varepsilon_{m}{ }^{(N)}(\hat{y})+O\left(g_{M+1}\right)
$$

where we denote the series of Eq. (23) as $H_{M}\left[E_{N}(\psi)\right]$. Thus

$$
H_{M}\left[E_{N}(\psi)\right]=\sum_{m=0}^{M} g_{m}(\epsilon) \mathcal{E}_{m}{ }^{(N)}(\hat{y}) .
$$

(c) The following equation is valid:

$$
E_{N}\left[H_{M}(\psi)\right]=H_{M}\left[E_{N}(\psi)\right] .
$$

In practice, for a given $N$ and $M, E_{N}\left[H_{M}(\psi)\right]$ would be obtained by taking the function $H_{M}(\psi)$ of Eq. (17), rewriting the $\hat{\psi}_{n}$ in the outer variable and expanding them on the basis of the outer limit, regrouping the terms according to the $\left\{G_{n}\right\}$ sequence, eliminating all terms of $o\left(G_{N}\right)$ and obtaining (if our above matching principle along with its assumptions are correct) and expression of the form given on the right-hand side of Eq. (22). $H_{M}\left[E_{N}(\psi)\right]$ would be obtained in an analogous way.

2.2 The inner expansion. Accepting the above assumptions 1 and 2 and assuming the validity of the matching principle, we proceed to seek solutions for the terms of the inner and outer expansions.

Setting $M=0$ in the inner expansion of (16), we insert it in Eqs. (14), take the inner limit and with full generality find that

$$
g_{0}(\epsilon)=\epsilon, \quad \hat{\psi}_{0}=\hat{y}=1+\hat{z} .
$$

The above procedure continued sequentially for subsequent $M>0$ results in the following conclusions.

The inner expansion of $\psi$ to $M+1$ terms, relative to the sequence of gauge functions

$$
\left\{g_{m}(\epsilon)\right\}=\left\{\epsilon^{m+1}\right\}, \quad(m=1,2, \cdots, M+1),
$$

is given by Eq. (16) if $\psi_{0}$ is given as in Eq. (26) and if subsequent $\psi_{n}$ s, obtained sequentially, satisfy

$$
\begin{aligned}
d^{2} \hat{\psi}_{1} / d \hat{y}^{2} & =(1 / \hat{y}-1) \equiv h_{1}(\hat{y}), \quad \hat{\psi}_{1}(1)=d \hat{\psi}_{1}(1) / d \hat{y}=0 ; \\
d^{2} \hat{\psi}_{m} / d \hat{y}^{2} & =-(1 / \hat{y})\left[(\hat{y}-1) d \hat{\psi}_{m-1} / d \hat{y}+\sum_{p=1}^{m-1} \hat{\psi}_{p} d^{2} \hat{\psi}_{m-p} / d \hat{y}^{2}\right] \equiv h_{m}(\hat{y}), \\
\hat{\psi}_{m}(1) & =d \hat{\psi}(1) / d \hat{y}=0, \quad m>1 ;
\end{aligned}
$$

provided the inner limit of (15) is restricted to $\hat{y}_{0}>0$. In other words, Eq. (16) is valid for all bounded $\hat{y}>0(\hat{z}>-1)$.

Besides the above solution for $\hat{\psi}_{0}$, three more of the $\hat{\psi}_{m}$ have been obtained. They are $\hat{\psi}_{1}=\hat{y}^{2} / 2+\hat{y} \ln \hat{y}+1 / 2$, 


$$
\begin{aligned}
& \hat{\psi}_{2}=\hat{\psi}^{3} / 12-3 \hat{y}^{2} / 4+(3 / 2) \hat{y} \ln \hat{y}-3 \hat{y} / 4+(1 / 2) \ln \hat{y}+17 / 12, \\
& \hat{\psi}_{3}=\hat{\psi}^{4} / 144-(1 / 12) \hat{y}^{3} \ln \hat{y}+7 \hat{y}^{3} / 72+(3 / 4) \hat{y}^{2} \ln \hat{y}-2 \hat{y}^{2}-(1 / 2) \hat{y} \ln ^{2} \hat{y} \\
&+(47 / 12) \hat{y} \ln \hat{y}-22 \hat{y} / 9+(5 / 3) \ln \hat{y}+607 / 144+1 /(8 \hat{y}) .
\end{aligned}
$$

These $\hat{\psi}_{n}, n=0,1,2,3$, will be eventually utilized in the determination of the first few terms of our outer expansion. With more work, any arbitrary number of subsequent $\hat{\psi}_{n}$ could also be obtained at this point. As pointed out by Van Dyke [6], this is the nature of singular perturbation solutions to initial-value problems. Without further effort it is apparent that all $h_{n}(\hat{y})$ and therefore all $\hat{\psi}_{n}$ are analytic functions of $\hat{y}$ in the entire complex $\hat{y}$ plane save for $\hat{y}=0$, at which point some and probably all $\hat{\psi}_{n}, n>0$ have an essential singularity. It is for this reason that we must restrict the inner limit, as stated below Eqs. (28), to $\hat{y}_{0}>0$. Furthermore, this singularity at $\hat{y}_{0}=0$ indicates that in the inner variables, and in the limit $\epsilon \downarrow 0$, the study of $\psi$ will require other singular perturbation expansions in the neighborhood of $\hat{y}=0$. As indicated earlier, such a study will not be reported in the present paper.

In view of the essentially complete state of our inner expansion for $\psi$, we now turn our attention to the problem of determining the outer expansion.

2.3 The first term of the outer expansion. Using the outer expansion of (19) with $N=0$ in Eq. (7), we take the outer limit, and find that without loss of generality three possibilities exist for an admissible $G_{0}$ and $\psi_{0}$, viz. (i) $1 / G_{0}=o(1)$ as $\epsilon \downarrow 0$ and $\psi_{0}=$ $C_{1}+C_{2} z$, where at least one of $C_{1}$ and $C_{2}$ is nonzero; (ii) $G_{0}=1$ and $\psi_{0}$ is a nontrivial solution of $\psi_{0} d^{2} \psi_{0} / d z^{2}+z \psi_{0}=0$; and (iii) $G_{0}=o(1)$ as $\epsilon \downarrow 0$ and $\psi_{0}=C_{1} \neq 0$. In order to deduce the correct result from these possibilities we use our matching principle for $M=N=0$. From (b) of this principle we immediately find that (i) is not a solution. From (b) and (c) we find that (iii) can also be dismissed. We therefore focus our attention on (ii) and we require $\psi_{0}$ to be a nontrivial solution of

$$
\psi_{0} d^{2} \psi_{0} / d z^{2}+z d \psi_{0} / d z=0
$$

Assuming $\psi_{0}$ to be an analytic function of $z$ in some fixed neighborhood of $z=0$, we can write $E_{0}(\psi)=\psi_{0}=\sum_{n=0}^{\infty} a_{n} z^{n}$. Rewriting $E_{0}(\psi)$ in the inner variable, taking the inner limit and eliminating all terms of $o\left(g_{M}\right)$ for arbitrary $M$, we find $H_{M}\left[E_{0}(\psi)\right]=$ $a_{0}+\sum_{m=0}^{M} g_{m} a_{m+1} \hat{z}^{m+1}$. Comparing to the required form of $H_{M}\left[E_{0}(\psi)\right]$ as per Eq. (24), it becomes clear that we must take $a_{0}=0$. Thus, for any nonnegative $M$ we have

$$
H_{M}\left[E_{0}(\psi)\right]=\sum_{m=0}^{M} g_{m} a_{m+1} \hat{z}^{m+1}=\sum_{m=0}^{M} a_{m+1} z^{m+1} .
$$

Taking $M=0$ in Eq. (17) and using our results for $\hat{\psi}_{0}$ as given in Eq. (26), we find $H_{0}(\psi)=\epsilon \hat{y}=\epsilon(\hat{z}+1)$. Rewriting $H_{0}(\psi)$ in outer variables, taking the outer limit and eliminating all terms of $o\left(G_{0}\right)$, we find

$$
E_{0}\left[H_{0}(\psi)\right]=z .
$$

Applying Eq. (25) of our matching principle for $M=N=0$ and using Eqs. (33) and (34), we conclude that $a_{1}=1$. With $a_{0}$ and $a_{1}$ in hand we finally summarize and establish the following results for $G_{0}(\epsilon)$ and $\psi_{0}(z)$.

It is consistent with our matching principle to take

$$
G_{0}(\epsilon)=1
$$


and to take $\psi_{0}(z)$ as satisfying

$$
\psi_{0} d^{2} \psi_{0} / d z^{2}+z d \psi_{0} / d z=0, \quad \psi_{0}(0)=0, \quad d \psi_{0}(0) / d z=1,
$$

where, in some neighborhood of $z=0$,

$$
\psi_{0}(z)=\sum_{n=1}^{\infty} a_{n} z^{n}
$$

Using Eq. (37) in Eqs. (36) yields the result

$$
\begin{aligned}
& a_{1}=1, \quad a_{2}=-1 / 2, \\
& a_{n}=-[n(n-1)]^{-1}\left[(n-1) a_{n-1}+\sum_{m=1}^{n-2}(n-m)(n-m-1) a_{m+1} a_{n-m}\right] .
\end{aligned}
$$

Besides obtaining these latter analytic results for $\psi_{0}$, we have also found $\psi_{0}$ from a numerical integration of Eqs. (36). From this we specifically find the result

$$
\psi_{0}(\infty)=0.714844 \cdots
$$

A plot of $\psi_{0}(z)$ is presented in Fig. 1 .

Before leaving the $G_{0}, \psi_{0}$ results, it is interesting to point out that with $\hat{\psi}_{1}, \hat{\psi}_{2}$, and $\hat{\psi}_{3}$ in hand, our matching principle can be immediately invoked in the form $E_{0}\left[H_{M}(\psi)\right]=$ $H_{M}\left[E_{0}(\psi)\right], M=1,2$, and 3 . In each of these three applications we obtain, happily, verification of our solutions for $a_{1}, a_{2}$, and $a_{3}$ as given in Eq. (38).

2.4 Governing equations for subsequent terms of the outer expansion and their homogeneous solution. With the results for $G_{0}$ and $\psi_{0}$ we can now proceed to study higher-

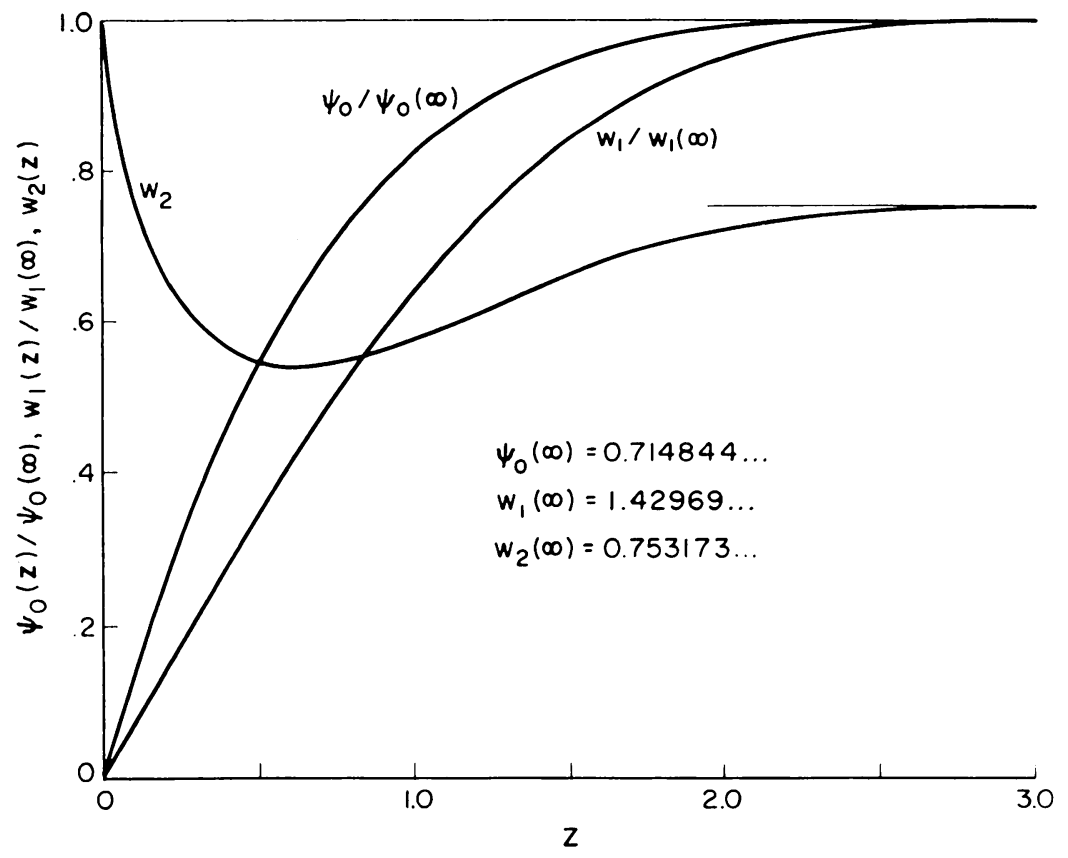

FIG. 1. Plots of $\psi_{0}(z), w_{1}(z)$ and $w_{2}(z)$. 
order terms in our outer expansions. Using the outer expansion of (19) in Eq. (7) sequentially with $N=1,2$, etc., we find that

$$
\begin{aligned}
\mathfrak{L}\left\{\psi_{n}\right\} & \equiv d^{2} \psi_{n} / d z^{2}+\left(z / \psi_{0}\right) d \psi_{n} / d z-\left(z d \psi_{0} / d z\right) \psi_{n} / \psi_{0}{ }^{2} \\
& =p_{n}(z), \quad n>0 .
\end{aligned}
$$

where $p_{1}=0$ and where, for example, the first three nonzero $p_{n}$ and corresponding $G_{n}$ will be given by

$$
\begin{aligned}
p_{m}= & -\left(\psi_{1} / \psi_{0}\right) d^{2} \psi_{1} / d z^{2}, \quad G_{m}=G_{1}{ }^{2} ; \\
p_{m+a}= & -\left[\psi_{1} d^{2} \psi_{2} / d z^{2}+\psi_{2} a^{2} \psi_{1} / d z^{2}\right] / \psi_{0}, \quad G_{m+a}=G_{1} G_{2} ; \\
p_{m+a+r}= & -\left[\psi_{1} d^{2} \psi_{3} / d z^{2}+\psi_{3} d^{2} \psi_{1} / d z^{2}\right] / \psi_{0}, \quad G_{m+a+r}=G_{1} G_{3}, \\
& \quad \text { if } G_{2}{ }^{2} /\left(G_{1} G_{3}\right)=o(1) \text { as } \epsilon \downarrow 0, \\
= & -\left(\psi_{2} / \psi_{0}\right) d^{2} \psi_{2} / d z^{2}, \quad G_{m+a+r}=G_{2}{ }^{2} \\
& \quad \text { if } G_{1} G_{3} / G_{2}{ }^{2}=o(1) \text { as } \epsilon \downarrow 0, \text { or } \\
= & -\left[\psi_{2} d^{2} \psi_{2} / d z^{2}+\psi_{1} d^{2} \psi_{3} / d z^{2}+\psi_{3} d^{2} \psi_{1} / d z^{2}\right] / \psi_{0}, \quad G_{m+a+r}=G_{2}{ }^{2} .
\end{aligned}
$$

Here, $m \geq 1, q \geq 1$ and $r \geq 1$ are (presently unknown) integers.

To study solutions for the $\psi_{n}$ we must first investigate solutions to the homogeneous equation

$$
\mathcal{L}\{W(z)\}=0 .
$$

According to our above knowledge of $\psi_{0}$, this linear homogeneous, second-order differential equation for $W(z)$ possesses a regular singular point at $z=0$. Moreover, using the well-known theory of such differential equations [7], we can find that independent solutions $w_{1}(z)$ and $w_{2}(z)$ to Eq. (42) are given by

$$
\begin{aligned}
& w_{1}(z)=\sum_{n=0}^{\infty} b_{n} z^{n+1} \\
& w_{2}(z)=w_{1}(z) \ln z+h(z)
\end{aligned}
$$

where $w_{1}(z)$ and $h(z)$ are analytic functions of $z$ in a neighborhood of $z=0$ (the same neighborhood of analyticity as $\psi_{0}$ ), i.e.

$$
h(z)=\sum_{n=0}^{\infty} c_{n} z^{n}
$$

and where, specifically, the first few $b_{n}$ and $c_{n}$ have been found to be

$$
\begin{aligned}
& b_{0}=1, \quad b_{1}=0, \quad b_{2}=-1 / 12, \quad b_{3}=-1 / 72, \cdots, \\
& c_{0}=1, \quad c_{1}=0, \quad c_{2}=-1 / 2, \quad c_{3}=1 / 24, \cdots \text {. }
\end{aligned}
$$

We have incorporated the above analytic results for $w_{1}(z)$ and $w_{2}(z)$ in obtaining these functions from numerical integrations. In particular, since $w_{1}(z)$ is analytic at $z=0$ and since, according to our results of Eq. (46), jt has known initial conditions, there was no particular difficulty in numerically integrating

$$
\mathfrak{L}\left\{w_{1}(z)\right\}=0, \quad w_{1}(0)=0, \quad d w_{1}(0) / d z=1
$$


simultaneously with the integration of the governing initial-value problem of Eqs. (36) for $\psi_{0}$. Furthermore, the function $w_{2}$, although poorly behaved at $z=0$, can easily be formed as per Eq. (44) from the analytic function $w_{1}$ and $h$. Indeed, from $\mathscr{L}\left\{w_{2}\right\}=0$, and from Eqs. (44)-(47) we find that $h$ satisfies

$$
\begin{gathered}
\mathscr{L}\{h\}=w_{1}\left(1 / z^{2}-1 / \psi_{0}\right)-(2 / z) d w_{1} / d z, \\
h(0)=1, \quad d h(0) / d z=0 .
\end{gathered}
$$

$w_{1}(z), h(z)$ and, as a consequence, $w_{2}(z)$ have been found (simultaneously with $\psi_{0}(z)$ ) by a numerical integration of the initial-value problems of Eqs. (36), (47) and (48). From this effort we specifically obtain the results

$$
w_{1}(\infty)=1.42969 \cdots, \quad w_{2}(\infty)=0.753172 \cdots .
$$

The functions $w_{1}(z)$ and $w_{2}(z)$ are plotted in Fig. 1. $h(z)$ is plotted in Fig. 2.

In view of all the above we conclude that

$$
\psi_{n}(z)=C_{1}{ }^{(n)} w_{1}(z)+C_{2}{ }^{(n)} w_{2}(z)+\Psi_{n}(z),
$$

where the $C_{1}{ }^{(n)}$ and $C_{2}{ }^{(n)}$ are constants to be determined (by our matching principle) and where $\Psi_{n}(z)$, a particular solution to $\mathscr{L}\left\{\Psi_{n}(z)\right\}=p_{n}(z)$ can be explicitly written as [7]

$$
\begin{aligned}
\Psi_{n}(z)= & -w_{1}(z) \int w_{2}(\xi) p_{n}(\xi) d \xi / \Delta\left[w_{1}(\xi), w_{2}(\xi)\right] \\
& +w_{2}(z) \int w_{1}(\xi) p_{n}(\xi) d \xi / \Delta\left[w_{1}(\xi), w_{2}(\xi)\right]
\end{aligned}
$$

where $\Delta\left[w_{1}(z), w_{2}(z)\right]=w_{1} d w_{1} / d z-w_{2} d w_{2} / d z=$ Wronskian. From the governing

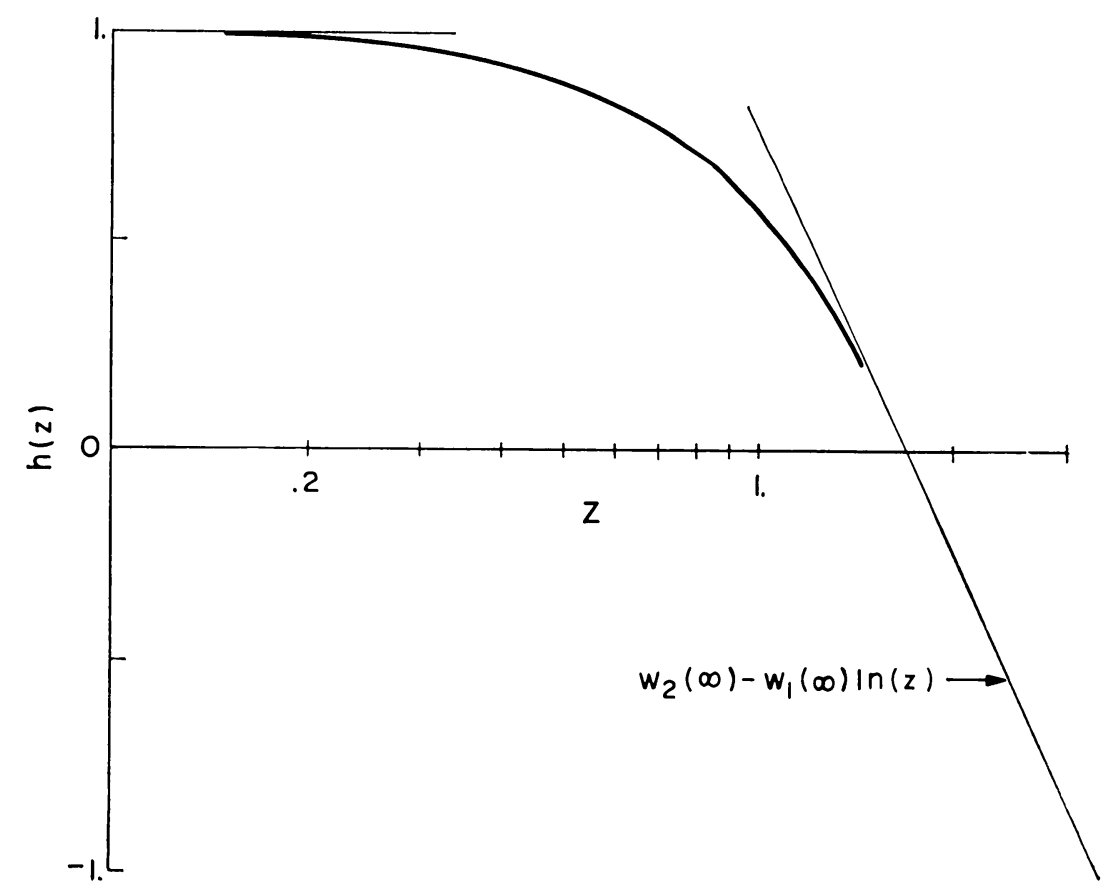

FIG. 2. Plot of $h(z)$. 
equation (42) for $w_{1}$ and $w_{2}$ it is a simple matter to deduce an explicit result for $\Delta$ and its reciprocal. Thus we have found

$$
\begin{aligned}
& \Delta=-d \psi_{0} / d z=-\sum_{n=1}^{\infty} n a_{n} z^{n-1}, \quad 1 / \Delta=\sum_{n=0}^{\infty} \delta_{n} z^{n} \\
& \delta_{0}=-1, \quad \delta_{n}=-\sum_{m=1}^{n}(m+1) a_{m+1} \delta_{n-m}, \quad n \geq 1 .
\end{aligned}
$$

$2.5 \mathrm{~A}$ solution attempt for $\psi_{1}(z)$. Since $p_{1}=0$, it is clear that $\psi_{1}$ is given by Eq. (50) where $\Psi_{1}=0$. With this knowledge we can now proceed to invoke our matching principle in order to establish both an appropriate $G_{1}$ and the values of $C_{1}{ }^{(1)}$ and $C_{2}{ }^{(1)}$.

From our above results we can write $E_{1}(\psi)=\psi_{0}+G_{1}\left[C_{1}{ }^{(1)} w_{1}+C_{2}{ }^{(1)} w_{2}\right]$. In an attempt to form $H_{M}\left[E_{1}(\psi)\right]$, we proceed to rewrite $E_{1}(\psi)$ in the inner variable, and take the inner limit. Then item (b) of our matching principle requires $C_{2}{ }^{(1)}=0$, and, with full generality, allows us to take $G_{1}=\epsilon^{a}$ where $q \geq 1$ is an integer.

We now proceed to study the various matchings $E_{1}\left[H_{M}(\psi)\right]=H_{M}\left[E_{1}(\psi)\right]$ in order to determine $q$ and $C_{1}{ }^{(1)}$. Starting with $M=0$ we take $H_{0}(\psi)$ as seen above Eq. (34), rewrite it in the outer variable and take the outer limit. It then becomes apparent that $q=1$ if item (a) of our matching principle is to be valid for $M=0, N=1$. Finally, in an attempt to impose item (c) of the matching principle we find that it is impossible to complete the match $E_{1}\left[H_{0}(\psi)\right]=H_{0}\left[E_{1}(\psi)\right]$ !

In a development similar to the above we have found that the various matchings involving $E_{1}\left[H_{M}(\psi)\right]=H_{M}\left[E_{1}(\psi)\right]$ are also not possible for $M=1$ and 2 , and are not likely for larger $M$. We therefore conclude that the matching principle is not valid for the combination $N=1$ and $M$ arbitrary. In this regard it is timely to mention that the success of the Van Dyke matching principle in every application for arbitrary $M$ and $N$ has been noted by Fraenkel to be less than universal [5].

2.6 The solution for $\psi_{1}(z)$ and $\psi_{2}(z)$. As mentioned earlier, our selection $G_{0}=1$ was with complete generality and, indeed, we still anticipate the possibility of evaluating higher-order terms of the outer expansion by using our matching principle. Since the principle was not applicable for $N=1$, we proceed to the case $N=2$, disregarding all tentative results mentioned in Sec. 2.5.

Whatever the correct order of $G_{1}(\epsilon)$, we tentatively assume that $G_{2}(\epsilon)$ is such that

$$
G_{1}^{2}(\epsilon)=o\left[G_{2}(\epsilon)\right] \quad \text { as } \quad \epsilon \downarrow 0 .
$$

Then, as per Eqs. (41), $p_{2}=0$ and $\psi_{1}$ and $\psi_{2}$ are given by Eq. (50) where $\Psi_{1}=\Psi_{2}=0$. Thus

$$
E_{2}(\psi)=\psi_{0}+G_{1}(\epsilon)\left[C_{1}^{(1)} w_{1}(z)+C_{2}{ }^{(1)} w_{2}(z)\right]+G_{2}(\epsilon)\left[C_{1}^{(2)} w_{1}(z)+C_{2}^{(2)} w_{2}(z)\right] .
$$

To form $H_{M}\left[E_{2}(\psi)\right]$ we rewrite $E_{2}(\psi)$ in the inner variable, take the inner limit and obtain the expression

$$
\begin{aligned}
\sum_{m=0}^{\infty} g_{m} a_{m+1} \hat{z}^{m+1}+ & C_{1}{ }^{(1)} \sum_{m=1}^{\infty} g_{m} G_{1} b_{m} \hat{z}^{m+1}+C_{1}{ }^{(2)} \sum_{m=0}^{\infty} g_{m} G_{2} b_{m} \hat{z}^{m+1} \\
+ & C_{2}{ }^{(1)}\left[G_{1}+\sum_{m=0}^{\infty} g_{m} G_{1}\left(b_{m} \ln \hat{z}+c_{m+1}\right) \hat{z}^{m+1}+\sum_{m=0}^{\infty}\left(g_{m} G_{1} \ln \epsilon\right) b_{m} \hat{z}^{m+1}\right] \\
+ & C_{2}^{(2)}\left[G_{2}+\sum_{m=0}^{\infty} g_{m} G_{2}\left(b_{m} \ln \hat{z}+c_{m+1}\right) \hat{z}^{m+1}+\sum_{m=0}^{\infty}\left(g_{m} G_{2} \ln \epsilon\right) b_{m} \hat{z}^{m+1}\right] .
\end{aligned}
$$


We now assume, for the moment, that $\epsilon=o\left[G_{1}(\epsilon)\right]$ and, of course, $G_{1}(\epsilon)=o\left[G_{0}(\epsilon)\right]=$ $o(1)$. Then, since $C_{2}{ }^{(1)} G_{1}(\epsilon)$, the largest term of the above expression, cannot be canceled by any other terms, and since it also cannot be ordered according to the $g_{m}$ (as would be required by (b) of our matching principle), it is clear that we must require $C_{2}{ }^{(1)}=0$. Furthermore, the terms of the coefficient of $C_{1}{ }^{(1)}$, which clearly cannot be ordered according to the $g_{m}$, can be canceled so as to give proper form as per (b) if we take $G_{1}=G_{2} \ln \epsilon$ and $C_{2}{ }^{(2)}=-C_{1}{ }^{(1)}$. Consistent with our original assumption of Eq. (53), further application of item (b) of the matching principle allows us to take $G_{2}=\epsilon$ without loss in generality and finally to take $C_{1}{ }^{(1)}$ and $C_{1}{ }^{(2)}$ to be arbitrary constants.

In view of the above, we have

$$
\begin{aligned}
H_{M}\left[E_{2}(\psi)\right]= & G_{0} z-C_{1}{ }^{(1)} G_{2}, \quad M=0 ; \\
= & G_{0}\left(\sum_{m=0}^{M} a_{m+1} z^{m+1}\right)+G_{1}\left(C_{1}{ }^{(1)} \sum_{m=1}^{M} b_{m-1} z^{m}\right) \\
& +G_{2}\left\{-C_{1}{ }^{(1)}+\sum_{m=0}^{M}\left[C_{1}{ }^{(2)} b_{m-1}-C_{1}{ }^{(1)}\left(c_{m}+b_{m-1} \ln z\right)\right] z^{m}\right\}, \quad M>0 ; \\
& C_{1}{ }^{(1)}, C_{1}{ }^{(2)} \text { are arbitrary constants, } G_{1}(\epsilon)=\epsilon \ln \epsilon, G_{2}(\epsilon)=\epsilon .
\end{aligned}
$$

With $G_{0}, G_{1}$ and $G_{2}$ we are now able to form

$$
E_{N}\left[H_{0}(\psi)\right]=z+\epsilon=G_{0}(\epsilon) z+G_{2}(\epsilon), \quad N \geq 2 .
$$

Then, using Eqs. (55) and (56) and requiring (c) of our matching principle to be satisfied for $M=0, N=2$, we obtain

$$
C_{1}^{(1)}=-1
$$

Similarly, with $M=1, N=2$ we find

$$
E_{2}\left[H_{1}(\psi)\right]=G_{0}(\epsilon)\left[z-z^{2} / 2\right]-z G_{1}(\epsilon)+G_{2}^{\prime}(\epsilon)[1-z+z \ln z] .
$$

We can, happily, complete the match $E_{2}\left[H_{1}(\psi)\right]=H_{1}\left[E_{2}(\psi)\right]$ with the use of Eqs. (55), (57) and (58) provided that

$$
C_{2}^{(1)}=-1
$$

Since $H_{2}(\psi)$ and $H_{3}(\psi)$ are also at our disposal, it has been verified that, when taking $M=1$ and $N=2$ or 3 , our matching principle is entirely consistent with the above results of this section which can be summarized as

$$
\begin{array}{ll}
G_{1}(\epsilon)=\epsilon \ln \epsilon, & G_{2}(\epsilon)=\epsilon \\
\psi_{1}(z)=-v_{1}(z), & \psi_{2}(z)=-w_{1}(z)+w_{2}(z),
\end{array}
$$

where $w_{1}$ and $w_{2}$ were determined in Scc. 2.4.

Although the presently available three-term outer expansion for $\psi$ (along with the inner expansion results of Sec. 2.2) will be adequate in studying the significant singular phenomenon under consideration, it would be an attractive feature of this investigation if further results could be obtained. In lieu of a general solution to the apparently intractable problem of obtaining results for all terms of the outer expansion, any indication, heuristic or otherwise, of answers to the following questions would be of interest in this regard: (i) What is the order of error in the available three-term outer expansion, i.e., 
what is $O\left(G_{3}\right)$ ? (ii) Will the matching principle continue to have application when nonzero particular solutions $\Psi_{n}$ are engaged in the determination of subsequent $\psi_{n}$ ? (iii) If the matching principle continues to have utility in the determination of any number of higher-order terms in the outer expansion of $\psi$, what general rule of application must be followed?

In the next section question (i) will be precisely answered and question (ii) will be partially answered (i.e., for the first three nonzero $\Psi_{n}(z)$ ). A heuristic answer to the last question will finally be presented.

2.7 Higher-order terms of the outer expansion. Having obtained the $\psi_{n}$ and appropriate $G_{n}$ for $n=0,1$, and 2, we now turn our attention to obtaining these functions for $n=3$. As it turns out, attempts to deduce such results by using the matching principle are futile in a sense similar to that discussed in Sec. 2.5. In particular, if we assume that ${G_{1}}^{2}=$ $\epsilon^{2} \ln ^{2} \epsilon=o\left(G_{3}\right), G_{3}=o\left(G_{2}\right)=o(\epsilon)$ (i.e., that $p_{3}=\Psi_{3}=0$ ), then one can show that no nontrivial solution for $\psi_{3}$ (as per Eq. (50)) which satisfies (b) of our matching principle is possible. If, alternatively, we assume, with complete generality, that $G_{3}=G_{1}{ }^{2}=\epsilon^{2} \ln ^{2} \epsilon$ (recall from Eq. (41) that $G_{3}$ can be no smaller) then, after deducing the appropriate particular solution $\Psi_{3}$, one can again find, in a similar way, that no solution for $\psi_{3}$ (as per Eq. (50)) will satisfy our matching principle.

In view of the above we conclude that the matching principle is not valid for $N=3$. If it is to be valid at all for determining subsequent terms in the outer expansion then it is clear from the above paragraph that some grouping of the next few terms must be determined together (e.g., as in our determination of $G_{1}$ and $\psi_{1}$ together with $G_{2}$ and $\psi_{2}$ ). It has been noted by Van Dyke ([6], p. 201) that logarithmic terms in $\epsilon$ multiplied by algebraic terms involving some power of $\epsilon$, in the type of expansions that are being considered here, are intimately related to purely algebraic terms containing the same power of the perturbation quantity, and that they must be regarded as together constituting a single step in the process of successive approximation. For the matching principle of Sec. 2.1 to be generally valid in our problem it appears that this may indeed be a strict requirement. Again, this was seen to be the case in our earlier determination of $G_{1}=\epsilon \ln \epsilon, \psi_{1}$ and $G_{2}=\epsilon, \psi_{2}$. Moreover, as indicated in Eq. (41), we are certain that terms of order ${G_{1}}^{2}=\epsilon^{2} \ln ^{2} \epsilon, G_{1} G_{2}=\epsilon^{2} \ln \epsilon$ and ${G_{2}}^{2}=\epsilon^{2}$ must eventually enter our outer expansion.

The above ideas suggest that we seek $\psi_{3}, \psi_{4}$, and $\psi_{5}$ together under the following assumptions:

$$
G_{2}(\epsilon)=\epsilon^{2} \ln ^{2} \epsilon, \quad G_{4}(\epsilon)=\epsilon^{2} \ln \epsilon, \quad G_{5}(\epsilon)=\epsilon^{2} .
$$

Then $\psi_{3}(z), \psi_{4}(z)$, and $\psi_{5}(z)$ are given by Eqs. (50) and (51) where, according to Eqs. (41),

$$
\begin{aligned}
& p_{3}(z)=-\left[\psi_{1} d^{2} \psi_{1} / d z^{2}\right] / \psi_{0}=-\left[\begin{array}{lll}
w_{1} & d^{2} w_{1} / d z^{2}
\end{array}\right] / \psi_{0}, \\
& p_{4}(z)=-\left[\psi_{1} d^{2} \psi_{2} / d z^{2}+\psi_{2} d^{2} \psi_{1} / d z^{2}\right] / \psi_{0} \\
& =2 p_{3}(z)+\left[w_{1} d^{2} w_{2} / d z^{2}+w_{2} d^{2} w_{1} / d z^{2}\right] / \psi_{0}, \\
& p_{5}(z)=-\left[\psi_{2} d^{2} \psi_{2} / d z^{2}\right] / \psi_{0}=-p_{3}(z)+p_{4}(z)-\left[w_{2} d^{2} w_{2} / d z^{2}\right] / \psi_{0} .
\end{aligned}
$$

In order to establish the validity of our matching principle for $N=5$ we will require the result of imposing the inner limit on the sum of the particular solutions, $G_{3} \Psi_{3}+$ $G_{4} \Psi_{4}+G_{5} \Psi_{5}$. To find this result we write this last expression with the $\Psi_{n}$ in their integral 
representations as per Eq. (51). We then rewrite the entire expression in the inner variable. Finally, with full use of the analytic results for $\psi_{0}, w_{1}$, and $h$ (i.e. their expansions about $z=0$ ) we perform the integrations and eventually establish the result: Under the inner limiting process,

$$
\sum_{n=3}^{5} G_{n} \Psi_{n}=H_{M}\left(\sum_{n=3}^{5} G_{n} \Psi_{n}\right)+O\left(g_{M+1}\right)
$$

where $H_{M}\left(\sum G_{n} \psi_{n}\right)$ is the inner expansion to (arbitrary) order $M$ of $\sum G_{n} \psi_{n}$, and is given by

$$
H_{M}\left(\sum_{n=3}^{5} G_{n} \Psi_{n}\right)=\sum_{m=0}^{M} g_{m}(\epsilon) \theta_{m}(\hat{z}) \hat{z}^{m-1}
$$

where $\theta_{0}(\hat{z})=0 ; \theta_{m}(\hat{z})=\alpha_{m}+\beta_{m} \ln \hat{z}+\gamma_{m} \ln ^{2} \hat{z}, m>0$, and where the following $\alpha_{m}$, $\beta_{m}$, and $\gamma_{m}$ have been determined:

$$
\begin{aligned}
& \alpha_{1}=\beta_{1}=1, \quad \alpha_{2}=\gamma_{1}=\gamma_{2}=\gamma_{3}=0, \\
& \alpha_{3}=-7 / 4, \quad \beta_{2}=3 / 2, \quad \beta_{3}=1 / 2 .
\end{aligned}
$$

In view of the above result we can now write $E_{5}(\psi)$ in the inner variable, take the inner limit, and establish that item (b) of our matching principle requires

$$
C_{1}{ }^{(3)}=C_{2}{ }^{(3)}=C_{2}{ }^{(4)}=0, \quad C_{1}^{(4)}=-C_{2}^{(5)} \text {. }
$$

Using these results, we can now form explicit expressions for all the $H_{M}\left[E_{5}(\psi)\right]$. We are now in a position to invoke item (c) of our matching principle for arbitrary $M$ and $N=5$. Taking $M=0$ results in an identity. Taking $M=1$ and then $M=2$ yields the results

$$
C_{1}{ }^{(4)}=0, \quad C_{1}{ }^{(5)}=-2 .
$$

Finally, a partial check of our results and of the validity of our matching principle for $N=5$ is in evidence when the condition $H_{3}\left[E_{5}(\psi)\right]=E_{5}\left[H_{3}(\psi)\right]$ is invoked and is found to yield an identity.

Having obtained the above results, it would be a straightforward task to complete the $\psi_{3}, \psi_{4}, \psi_{5}$ solutions as per Eq. (50) by evaluating the $\Psi_{3}, \Psi_{4}, \Psi_{5}$, say, by a numerical integration of their governing equations. The initial conditions of these $\Psi_{n}$ functions along with their general behavior near $z=0$ would be required in order to proceed with such integrations. These could be obtained from the solution forms of Eq. (51) with the use of the known behavior of $\psi_{0}, w_{1}, w_{2}$ and $\Delta$ near $z=0$.

In summary, the first two of the questions posed at the end of Sec. 2.6 have been answered to the pledged degree of fullness. In answer to the last of these questions, it appears that our matching principle may be valid for every $N$ such that

$$
N=M+M(M+1) / 2 \quad M=0,1, \cdots
$$

where for every such $N$ and corresponding $M$ the generated $G_{n}$ s to be used in the match can be taken as

$$
\epsilon^{M} \ln ^{M}(\epsilon), \epsilon^{M} \ln ^{M-1}(\epsilon), \cdots, \epsilon^{M} .
$$

2.8 Composite expansions for $\psi$. At this point we are in possession of early terms of expansions for $\psi$ which are valid either under the inner limit or the outer limit. A 
single expansion, or sequence of expansions of increasing accuracy, valid under both the inner and outer limit, would be of considerable value in the actual representation and computation of this function. Such expansions, constructed from our $E_{N}(\psi), H_{M}(\psi)$, and $E_{N}\left[H_{M}(\psi)\right]$, can be conveniently given by [6]

$$
\psi^{[N, M]}=E_{N}(\psi) H_{M}(\psi) / E_{N}\left[H_{M}(\psi)\right]
$$

for every $N$ where our matching principle was applicable. Indeed, from all that has preceded it follows that for every such $N$

$$
\psi=\psi^{[N, M]}+ \begin{cases}0\left(g_{M+1}\right) & \text { under the inner limit, } \\ 0\left(G_{N+1}\right) & \text { under the outer limit. }\end{cases}
$$

In view of the above we can construct the following two successively more accurate uniform approximations for $\psi$ :

$$
\begin{aligned}
\psi(z, \epsilon)^{[0,0]}= & \psi_{0}(z)[\epsilon / z+1] \\
\psi(z, \epsilon)^{[2,1]}= & {\left[\psi_{0}(z)+\epsilon \ln \epsilon \psi_{1}(z)+\epsilon \psi_{2}(z)\right] } \\
& . \frac{\left[z-z^{2} / 2+\epsilon(1-z)+\epsilon^{2}(1+z / \epsilon) \ln (1+z / \epsilon)\right]}{\left[z-z^{2} / 2+\epsilon(1-z+z \ln z)-z \epsilon \ln \epsilon\right]} .
\end{aligned}
$$

In the next section two uniformly valid successive approximations for $\phi$ will be obtained from the above composite expansion estimates for $\psi$ by using the transformation of (9).

3. The solution for $\phi(\eta), 0<\phi_{s} \leq 1$.

3.1 The relationship between $\phi_{s}$ and $\zeta$. From (9) it is clear that $\epsilon=\phi_{s} \psi(\infty ; \epsilon)$. Using this and Eq. (70), it is possible to determine the existence of functions $\epsilon^{[N, M]}$ such that

$$
\lim _{\phi_{s} \downarrow 0} \epsilon=\epsilon^{[N, M]}+0\left[\phi_{s} G_{N+1}\left(\phi_{s}\right)\right] ; \quad \epsilon^{[N, M]} / \phi_{s}=\sum_{n=0}^{N} K_{n} G_{N+1}\left(\phi_{s}\right) .
$$

By using the results of Sec. 2 it follows that

$$
\begin{aligned}
\epsilon^{[0,0]} / \phi_{s} & =\psi_{0}(\infty) ; \\
\epsilon^{(2,1]} / \phi_{s} & =\psi_{0}(\infty)\left\{1+\psi_{1}(\infty) \phi_{s} \ln \phi_{s}+\left[\psi_{1}(\infty) \ln \psi_{0}(\infty)+\psi_{2}(\infty)\right] \phi_{s}\right\} .
\end{aligned}
$$

Furthermore, since $\zeta=\left[\phi_{s} / \epsilon\right]^{1 / 2}$ we can use the above to obtain the following $\zeta, \phi_{s}$ relationships:

$$
\begin{aligned}
\lim _{\phi_{s} \backslash 0} \zeta=\zeta_{c}+ & 0\left(\phi_{s} \ln \phi_{s}\right), \quad \zeta_{c}=\psi_{0}{ }^{-1 / 2}(\infty)=1.182754 \ldots ; \\
\lim _{\phi_{s} \backslash 0} 2\left[1-\zeta / \zeta_{c}\right]= & \psi_{1}(\infty) \phi_{s} \ln \phi_{s}+\left[\psi_{1}(\infty) \ln \psi_{0}(\infty)+\psi_{2}(\infty)\right] \phi_{s} \\
& +0\left(\phi_{s}{ }^{2} \ln ^{2} \phi_{s}\right) \\
= & (-1.42969 \ldots) \phi_{s} \ln \phi_{s}+(-.19659 \ldots) \phi_{s} \\
& +0\left(\phi_{s}{ }^{2} \ln ^{2} \phi_{s}\right) .
\end{aligned}
$$

Thus, the dramatic result is obtained that arbitrarily small imposed surface conductivity results in a dimensionless amplitude of heat transfer which approaches a fixed bounded value, $\zeta_{c}$. By using Eq. (75), $\phi_{s}$ has been plotted as a function of $1-\zeta / \zeta_{c}$ in Fig. 3. Included in Fig. 3 is, further, a similar plot determined from several numerical in- 


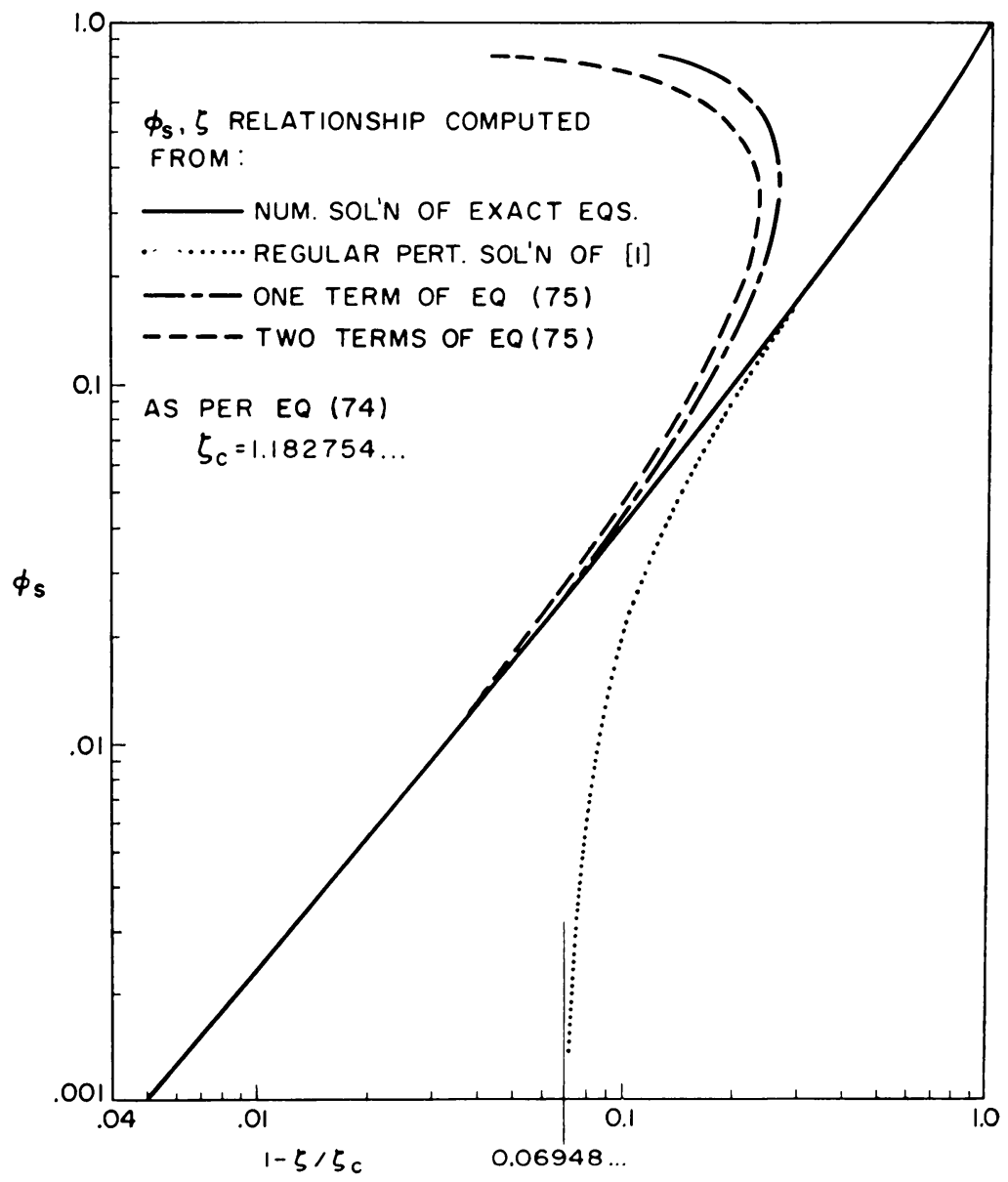

FIG. 3. The relationship between $\phi$ and $\zeta$.

tegrations of the exact initial-value problem, Eqs. (7)-(8). A final estimate for the $\phi_{s}, \zeta$ dependence, also plotted in Fig. 3, has been obtained from the four-term regular perturbation solution approximation of Eq. (6) (i.e., where the series has been truncated after three terms [1]).

The data presented in Fig. 3 illustrate the dependence of $\phi_{s}$ on $\zeta$ for all $0<\phi_{s} \leq 1$ or $0 \leq \zeta<\zeta_{c}$. While this figure can be considered to be a working plot, data of particular interest may be reproduced from the most judicious calculation indicated. In this regard it is of interest to note that the one-term estimate for $\left[1-\zeta / \zeta_{c}\right]$, available from Eq. (75) and accurate to $O\left(\phi_{s}\right)$, always appears to be superior to the two-term estimate.

3.2 The solution for $\phi\left(\eta ; \phi_{s}\right)$. From (9) it follows that $\phi=\psi \phi_{s} / \epsilon$. Using (70) and (72) in this, and defining (since $1 / \zeta=\left(\epsilon / \phi_{s}\right)^{1 / 2}$ and $\left.z=\eta / \zeta\right)$

$$
z^{[N, M]} \equiv \eta\left(\epsilon^{[N, M]} / \phi_{s}\right)^{1 / 2},
$$

we can show that

$$
\phi\left(\eta ; \phi_{s}\right)=\phi_{s} \psi^{[N, M]}\left(z^{[N, M]}, \epsilon^{[N, M]}\right) / \epsilon^{[N, M]}+\left\{\begin{array}{l}
0\left[\phi_{s} G_{N+1}\left(\phi_{s}\right)\right] \\
0\left[\ln \phi_{s} G_{N+1}\left(\phi_{s}\right)\right]
\end{array} .\right.
$$


Here upper and lower error estimates are for the inner and outer limit, respectively (where $\eta \psi_{0}{ }^{1 / 2}(\infty)$ and $\phi_{s} \psi_{0}(\infty)$ replace $\epsilon$ and $z$, respectively, in our previous definitions of these limits). Setting $N=M=0$ in Eq. (77) and using Eq. (71), we obtain

$$
\phi\left(\eta, \phi_{s}\right)=\psi_{0}\left[\eta \psi_{0}{ }^{1 / 2}(\infty)\right]\left[\phi_{s} \psi_{0}{ }^{1 / 2}(\infty) / \eta+1\right] / \psi_{0}(\infty)+0\left\{\begin{array}{l}
\phi_{s}{ }^{2} \ln \phi_{s} \\
\phi_{s} \ln ^{2} \phi s
\end{array}\right.
$$

A more refined estimate for $\phi$ is also available to us by setting $N=2, M=1$ in Eq. (77) and using our result for $\psi^{[2,1]}$ of Eq. (71).

The $\phi$ field has been computed and compared for different values of $\phi_{s}$ using the two available estimates of Eq. (77), numerical integrations of the exact equations for $\psi$ and, finally, the available four-term approximation of the regular perturbation solution for $\phi$. The results are presented in Fig. 4.

From Fig. 4 it appears that there is no practical advantage in using the composite expansion estimate which uses $\psi^{[2,1]}$ over the much simpler estimate of Eq. (78). Using this latter estimate, an error of less than $5 \%$ in $\phi$ is guaranteed for $\phi_{s} \leq .0243$ throughout the entire range $0 \leq \eta<\infty$. (The error is reduced to a maximum of $0.5 \%$ with the $\psi^{[2,1]}$ estimate.) For $1 \geq \phi_{s} \geq .0243$ the four-term regular perturbation approximation yields a solution with an error of less than $3 \%$ in $\phi$ for all $0 \leq \eta<\infty$. It has been finally, and most dramatically, deduced that this latter regular perturbation approximation yields an estimate which has an error of less than 8 percent in $\phi$ for all $0<\phi_{s} \leq 1$ in the entire

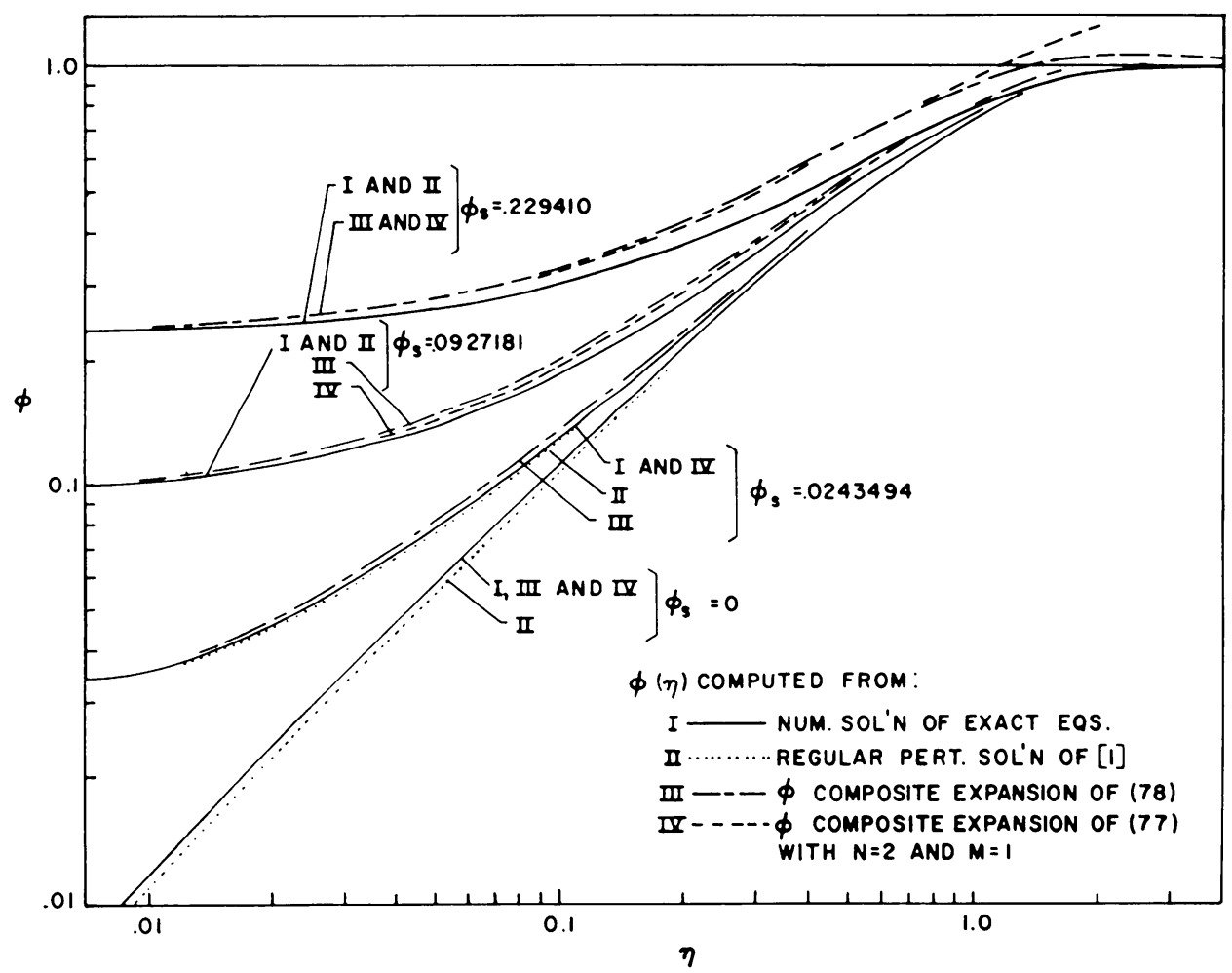

FIG. 4. Plot of $\phi$ as a function of $\eta$ with $\phi_{s}$ as a parameter. 
range $0 \leq \eta<\infty$. We have therefore shown that this estimate together with the previously deduced $\phi_{s}, \zeta$ relationship comprises a useful complete solution to the boundaryvalue problem that has been considered in this investigation.

4. Summary and conclusions. An approximate solution to the heated halfspace problem introduced in Sec. 1.1 and 1.2 has been obtained. In particular, this work, which has studied the behavior of $\phi$ under the surface conductivity limit $\phi_{s} \downarrow 0$, has resulted in a solution for the relationship between $\phi_{s}$ and $\zeta$ (see Sec. 3.1). Such a result was obtained by solving an auxiliary problem for $\psi$, introduced in Sec. 1.3, by Van Dyke's method of matched asymptotic solutions. It was concluded that the limit $\phi_{s} \downarrow 0$ corresponds to the limit $\zeta \uparrow \zeta_{c}=1.182754 \cdots$. Further, a solution for $\phi$ uniformly valid for all $0 \leq \eta$ as $\phi_{s} \downarrow 0$ has been obtained from the above-mentioned solution for $\psi$ (see Sec. 3.2). By using this solution, it has finally been shown that (for calculating the $\phi$ field, given $\phi_{s}$ ) the range of utility of a four-term truncated regular perturbation solution for $\phi$ can be extended to $0<\phi_{s} \leq 3.70[1]$ provided $8 \%$ errors in $\phi$ are acceptable. (The accuracy of this representation in the range $1 \leq \phi_{s} \leq 3.70$ was shown in [1].)

For materials with essentially constant $\rho C$ and a conductivity variation which can be accurately modeled as per Eq. (1) within a useful temperature range, the results obtained here and in [1] allow for simple estimates of the solution to the heating problem considered. When this temperature range includes the point of material melting or sublimation, etc., the results of our solution would yield a bound on the "amplitude" of $H=h t^{-1 / 2}$ type of surface heating within which the integrity of the surface woul $\downarrow$ be assured and outside of which the degradation of the material surface would be guaranteed. The results of this work would also provide useful temperature or heat-transfer bounds in heated, variable-conductivity material types other than those considered here. It is finally anticipated that the solution provided herein will find appropriate application in understanding other concentration-dependent diffusion phenomena.

\section{REFERENCES}

[1] L. Y. Cooper, Constant temperature at the surface of an initially uniform temperature, variable conductivity half space, J. Heat Trans. 93, 55-60 (1971)

[2] C. Wagner, On the solution to diffusion problems involving concentration-dependent diffusion coefficients, J. Metals 4, 91-96 (1952)

[3] J. Crank, Mathematics of diffusion, Oxford (1957)

[4] W. R. Gardner and M. S. Mayhugh, Solutions and tests of the diffusion equation for the movement of water in soil, Soil Sci. Soc. Am. Proc. 22, 197-201 (19.58)

[5] L. E. Fraenkel, On the method of matched asymptotic expansion-Part I: A matching principle, ProcCamb. Phil. Soc. 65, 209-231 (1969)

[6] M. Van Dyke, Perturbation methods in fuid mechanics, Academic Press (1964)

[7] G. F. Carrier, M. Krook, and C. E. Pearson, Functions of a complex variable, McGraw-Hill (1966) 